\title{
Static coarsening of titanium alloys in single field by cellular automaton model considering solute drag and anisotropic mobility of grain boundaries
}

\author{
WU Chuan, YANG He*, LI HongWei \& FAN XiaoGuang \\ State Key Laboratory of Solidification Processing, School of Materials Science and Engineering, Northwestern Polytechnical University, Xi'an \\ 710072, China
}

Received September 6, 2011; accepted November 22, 2011

\begin{abstract}
Static coarsening is an important physical phenomenon that influences microstructural evolution and mechanical properties. How to simulate this process effectively has become an important topic which needs to be dealt with. In this paper, a new cellular automaton (CA) model, which considers the effect of solute drag and anisotropic mobility of grain boundaries, was developed to simulate static grain coarsening of titanium alloys in the beta-phase field. To describe the effect of the drag caused by different solute atoms on coarsening, their diffusion velocities in beta titanium were estimated relative to that of titanium atoms (Ti). A formula was proposed to quantitatively describe the relationship of the diffusion velocity of Ti to that of solute atoms; factors influencing the diffusion velocity such as solute atom radius, mass, and lattice type were considered. The anisotropic mobility of grain boundaries was represented by the parameter $c_{0}$, which was set to 1 for a fully anisotropic effect. These equations were then implemented into the CA scheme to model the static coarsening of titanium alloys Ti-6Al-4V, Ti17 (Ti-5Al-4Mo-4Cr-2Sn-2Zr, wt\%), TG6 (Ti-5.8Al-4.0Sn-4.0Zr-0.7Nb-1.5Ta-0.4Si-0.06C, wt\%) and TA15 (Ti-6Al-2Zr-1Mo-1V, wt\%) in the beta field. The predicted results, including coarsening kinetics and microstructural evolution, were in good agreement with experimental results. Finally, the effects of time, temperature, and chemical composition on grain coarsening and the limitations of the model were discussed.
\end{abstract}

static grain coarsening, cellular automaton model, solute drag, anisotropic mobility, titanium alloys

Citation: Wu C, Yang $\mathrm{H}, \mathrm{Li} \mathrm{H} \mathrm{W}$, et al. Static coarsening of titanium alloys in single field by cellular automaton model considering solute drag and anisotropic mobility of grain boundaries. Chin Sci Bull, 2012, 57: 1473-1482, doi: 10.1007/s11434-012-5002-9

The mechanical properties of titanium alloys such as the creep resistance, fracture toughness and crack propagation resistance are determined by their grain sizes in the beta phase field [1,2]. However, the grains are prone to static coarsening when held in the beta phase field because of the structural characteristics of beta-titanium and relatively high stacking fault energy at high temperatures [3]; this results in a marked decrease in the mechanical properties of final products. Therefore, it is necessary to understand and to control static coarsening of titanium alloys in a single phase field to obtain the expected mechanical properties.

Previously, many experimental investigations have fo-

*Corresponding author (email: yanghe@nwpu.edu.cn) cused on the static coarsening behaviors of titanium alloys in the beta phase field. Ivasishin et al. [4,5] investigated the effect of texture evolution on the values of the static coarsening exponent $n$ and activation energy for Ti- $6 \mathrm{Al}-4 \mathrm{~V}$ alloy in the beta field. They found values of $n$ ranging from 0.22 to 0.31 at different temperatures, but there were marked deviations attributed to different materials and experiments when the results were compared with those of others. Recently, Semiatin et al. [6-8] investigated the static grain coarsening of Ti-6Al-4V alloy via a series of heat treatments at typical temperatures. They found that the coarsening process of primary alpha particles was controlled by the volume diffusion of solute elements at lower temperatures and the growth exponent was equal to 0.33 . 
Similarly, Bradley et al. [9] and Cotrina et al. [10] analyzed coarsening kinetics via experimental data from optical observations.

Recently, various numerical simulation techniques such as Potts-Monte Carlo models (MC) [11-13], phase field models $[14,15]$ and cellular automaton models (CA) [16-18] have been gradually used to model the microstructure evolution on the mesoscale [19]. The CA methods have been widely used in these investigations, and have some advantages [20]. Geiger et al. [21] investigated static coarsening by CA modeling and discussed the effects of orientation difference, activation energy, and boundary energy on coarsening. Subsequently, Raghavan et al. [22,23] and Kugler et al. [24] simulated the topological and kinetic features of grain coarsening in polycrystalline materials. However, most of these models neglected the effect of solute drag and the anisotropic mobility of grain boundaries on the microstructural evolution during the coarsening process [15]. The predicted results, including coarsening kinetics and topological evolution, generally deviated from the experimental results.

Solute drag and anisotropic mobility greatly influence the coarsening process. The diffusion velocity of solute atoms in the matrix is influenced by their radius, mass, and lattice type. If the diffusion velocity is substantially less than that of matrix, the solute atoms on the grain boundaries will retard the coarsening process; the drag effect on coarsening kinetics in this case is obvious. Because of the different orientations of each grain, the grain mobility is different from different grains, which also exerts an important influence on grain coarsening. However, most previous CA models for grain coarsening ignore the effects of solute drag and anisotropic mobility, which leads to a difference between simulated and experimental coarsening kinetics. Therefore, the objective of the present paper was to simulate grain coarsening by CA models taking into account solute drag and anisotropic mobility. Firstly, a formula was proposed to quantitatively describe the relationship between the diffusion velocities of $\mathrm{Ti}$ and solute atoms, which was then used to calculate the effect of solute drag. Secondly, a parameter representing the anisotropy of the grain boundary mobility was introduced. Finally, these equations were implemented into a CA scheme and the CA models were applied to simulate the grain coarsening; the predicted results were compared with the experimental measurements.

\section{Cellular automaton model}

\subsection{Static grain coarsening models}

Static coarsening is a thermally activated process that has been widely studied in the domain of carbon-steel alloys [25]. Usually it takes place by the jumping of atoms from one grain to another and the migration of grain boundaries. Atoms located in the grain boundaries with enough high energy might overcome the energy barriers to change into a new state [26]. Similarly, in CA models, the cells of boundaries transfer from one grain to another with probability $p$. According to the Maxwell-Boltzmann distribution law, the probability $p$ is determined by:

$$
\begin{gathered}
p=\frac{\Delta Q}{\Delta Q_{\max }} \Delta Q>0, \\
p=\frac{\exp \left(-\frac{Q_{b}}{R T}\right)}{\exp \left(-\frac{Q_{b}}{R T}\right)+\exp \left(-\frac{E}{R T}\right)} \Delta Q \leqslant 0,
\end{gathered}
$$

where $\Delta Q$ is the difference between the total energy of cell $i$ and self-diffusion activation energy $Q_{b}$. The total energy of each cell consists of systemic thermal energy $E^{t}$, and total boundary energy $E^{b}$. The thermal energy of cell $i$ is expressed as [27]:

$$
E_{i}^{t}=-R T \ln r,
$$

where $r(0<r<1)$ is a random number produced by the computer program, and $R$ and $T$ have their universal meanings. The boundary energy, $\gamma_{i j}$, between two neighboring cells $i$ and $j$ (dependent on misorientation) is assumed to follow the Read-Shockley equation [28]:

$$
\gamma_{i j}=\left\{\begin{array}{cc}
\gamma_{m}, & \theta_{i j} \geqslant \theta_{m} \\
\gamma_{i j}=\gamma_{m} \frac{\theta_{i j}}{\theta_{m}}\left[1-\ln \left(\frac{\theta_{i j}}{\theta_{m}}\right)\right], & \theta_{i j}<\theta_{m},
\end{array}\right.
$$

where $\theta_{m}$ is the misorientation for high-angle grain boundaries, usually set as $15^{\circ}$ and $\theta_{i j}$ is the misorientation angle between two adjacent cells $i$ and $j$; $\gamma_{m}$ is the boundary energy of the high-angle grain boundary, which is directly calculated by [29]:

$$
\gamma_{m}=\frac{\mu b \theta_{m}}{4 \pi(1-v)},
$$

where $\mu$ is the shear modulus of material at a specific temperature, $b$ is the magnitude of Burgers vector, $v$ is Poisson's ratio. The total boundary energy of cell $i$ is expressed as follows:

$$
E_{i}^{b}=\sum_{j}^{N} \gamma_{i j},
$$

where $N$ is the number of nearest neighbors of cell $i$ and is set to 4 according to the neighboring rule in this paper.

The migration of the grain boundaries is regarded as curvature-driven boundary migration, in which the driving force is supplied by the difference in total energy between two neighboring grains (cells). Thus, the velocity of the coarsening grain boundaries, $v$, is expressed as follows:

$$
v=m f,
$$

where $m$ and $f$ are the mobility of grain boundaries and driving force exerted on coarsening grain boundaries, respectively. The driving force $f_{i j}$ is expressed by:

$$
f_{i j}=\Delta E / R_{i j},
$$

where $\Delta E$ is the difference in total energy between two 
neighboring cells $i$ and $j, R_{i j}$ is the radius of the curvature of boundary located by cell $j$ adjacent to located by cell $i$. The growth distance $l$ of cell $i$ at its boundary towards one of the neighboring cells $j$ during the time increment $\Delta t$ is calculated by:

$$
l_{i j}(t+\Delta t)=l_{i j}(t)+v_{i j} \Delta t,
$$

where $l_{i j}(t)$ represents the growth distance of a cell at time $t$, the initial value is set to zero. $l_{i j}(t+\Delta t)$ is the growth distance at time $t+\Delta t$. Kugler et al. [30] gave the definition of time increment $\Delta t$ as the ratio of grid size, $d$, to the maximum grain boundary velocity, $v_{\max }$. According to eqs. (6)-(8), the maximum velocity is achieved when boundary energy $\gamma_{i j}$ and grain radius $R_{i j}$ are taken as $\gamma_{m}$ and initial value $R_{0}$, respectively. Thus the time increment $\Delta t$ is expressed by:

$$
\Delta t=C(T) \frac{d}{v_{\max }}=C(T) \frac{d \cdot R_{0}}{m\left(\gamma_{m}+E_{i j}^{t}\right)},
$$

where $d$ is grid size, $R_{0}$ is grain size of initial microstructure, and $C(T)$ is a constant related to temperature.

\subsection{Quantitative description of solute drag}

The concentration of solute atoms around the grain boundaries is relatively larger than that in the grain interior because of the structural defects at the grain boundaries. Because of the existing concentration gradient, the solute atoms on grain boundaries tend to diffuse; simultaneously, the diffusion of $\mathrm{Ti}$ (grain boundary migration) takes place during coarsening. If the diffusion velocity of solute atoms is smaller than that of $\mathrm{Ti}$ (grain boundary migration), the solute atoms will retard the movement of the boundaries. There are a variety of alloying elements in titanium, the major ones include the alpha-stabilizing elements, such as aluminum ( $\mathrm{Al}$ ), and beta-stabilizing elements, such as vanadium (V), molybdenum (Mo) and tin (Sn). The diffusion velocities for these solute atoms in beta titanium varies with the atom radius, mass and lattice type. Therefore, it is impossible and unrealistic to calculate the diffusion velocity of each type of solute atom in beta titanium. To quantitatively describe the effect of solute drag, the diffusion velocity of different types of solute atoms is related to that of Ti. For this purpose, a formula was proposed to describe the relationship between the diffusion velocities of solute atoms and Ti. It is assumed that the diffusion velocity ratio is inversely proportional to the radius and mass ratios of the solute atom to $\mathrm{Ti}$, leading to:

$$
\frac{v_{i}}{v_{\mathrm{Ti}}}=\frac{h}{\chi_{1} \cdot \chi_{2}},
$$

where $v_{i}$ and $v_{\mathrm{Ti}}$ are the diffusion velocity of solute atoms and titanium atoms, respectively. $h$ is a parameter representing the effect of the lattice type of the solute atoms on coarsening. If the lattice type of the solute atoms is the same as that of beta titanium (as occurs for V, Mo and $\mathrm{Sn}$ ), $h$ is set to 1 , otherwise, its value is less than $1 . \chi_{1}$ and $\chi_{2}$ are the atomic radius and mass ratios of the solute to $\mathrm{Ti}$, respectively:

$$
\begin{aligned}
& \chi_{1}=\frac{R_{i}}{R_{\mathrm{Ti}}}, \\
& \chi_{2}=\frac{m_{i}}{m_{\mathrm{Ti}}},
\end{aligned}
$$

where $R_{i}$ is the radius of the solute atom, $R_{\mathrm{Ti}}$ the radius of the titanium atom, $m_{i}$ the mass of the solute atom and $m_{\mathrm{Ti}}$ the mass of the titanium atom. If the value of $v_{i} / v_{\mathrm{Ti}}$ is larger than 1 , the diffusion velocity of the solute atoms is larger than that of $\mathrm{Ti}$, which means the solute drag is negligible. Otherwise, the diffusion velocity of Ti will be retarded by the solute atoms. To incorporate the solute drag effect into $\mathrm{CA}$ models requires the formulation of $\mathrm{CA}$ transfer rules, which is discussed in Section 1.4.

\subsection{Anisotropic mobility}

In conventional CA models, the assumption of misorientation-independent grain boundary mobility was made, so that the predicted topology features (size and side distribution) did not reproduce the log-normal distribution observed experimentally-instead the simulated topology distribution of grains appeared more left-skewed [31]. In fact, the grain boundary mobility is dependent on the misorientation and becomes anisotropic. Therefore, we introduced a parameter $c_{0}$ (eq. (13)) to represent the effect of anisotropic mobility on grain coarsening. The mobility of grain boundaries $m_{i j}$ between two neighboring cells, $i$ and $j$, is calculated by [32]:

$$
m_{i j}=m_{0}\left\{1-c_{0} \cdot \exp \left[-\left(\frac{\theta_{i j}}{10^{\circ}}\right)^{3}\right]\right\},
$$

where $m_{0}$, a constant, is the mobility of high-angle boundaries. The parameter $c_{0}$ is introduced to take into consideration anisotropic grain boundary mobility, and is set to 0 and 1 for the isotropic and anisotropic cases, respectively.

\subsection{CA simulation steps for static grain coarsening}

In the present simulation, the initial microstructure was obtained by running the CA program with conditions of homogenous site-saturated nucleation and equiaxed growth. The state variables including the grain orientation variable, energy variables (total, thermal and boundary), diffusion velocity variable, location variable and property variable are defined for each cell. The cell size is defined according to the average grain size of the initial microstructures.

Values of the orientation variable, ranging from 0 to 180 , are automatically assigned by the computer program before CA simulation. Energy and velocity variables are calculated in each step of the CA simulation and assigned to each cell. The location variable is used to specify whether a cell is 
located in the grain interior (represented by 1) or on a boundary (represented by 0 ). The property variable is used to specify whether a cell belongs to Ti (represented by 0 ) or other types of solute atoms (represented by an integer larger than 0 ).

In each simulation step, the state variables are updated according to the $\mathrm{CA}$ rules and the microstructural evolution of CA modeling proceeds. A single cycle through the grain coarsening loop consists of cell jumping and migration of grain boundaries, as illustrated in Figure 1.

(1) Cell jumping. The cells located on the boundaries are selected. According to eqs. (2)-(4), the thermal and boundary energy variables for these cells are calculated and used to obtain the total energy (eq. (5)). Then the total energy of each cell on the boundaries is compared with the diffusion activation energy; if the difference between them is larger than 0 , the cell jumps with a transition probability determined by eq. (1a). Otherwise, the transition probability is calculated by eq. (1b). At the same time, a random number $r(0<r<1)$ is generated for each boundary cell. If the transition probability is no less than the random number, these cells start to coarsen.

(2) Migration of grain boundary. For each jumping cell, the property variable of its neighboring cell which it grows towards is evaluated during the coarsening process. If the neighboring cell is occupied by the solute cell representing solute atoms, the movement of the jumping cell might be dragged by the solute cell. To incorporate the drag effect into CA models, the CA transfer rules are formulated as follows: the diffusion velocity of solute atoms, relative to that of Ti (eq. (10)), will be compared with that of Ti; if the difference is less than zero, the solute atoms will retard the movement of the boundary cell and the migration velocity of Ti will be decreased by the diffusion velocity ratio; otherwise, the velocity stays the same.

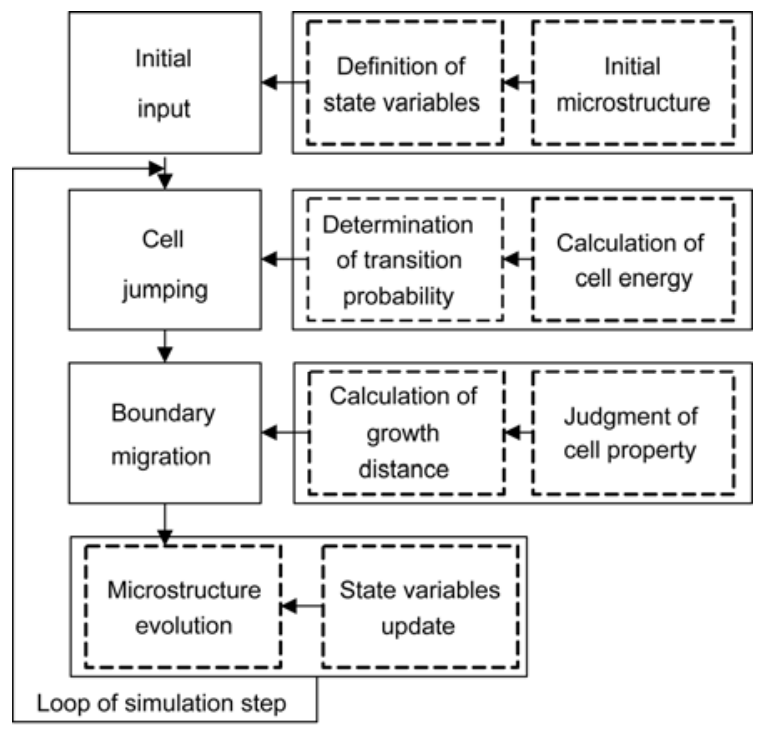

Figure 1 Coarsening simulation step.
The growth distance of a jumping cell at time $t$ could be calculated by eq. (8). The ratio of growth distance to grid size determines whether the cell can take place in coarsening or not. If the value is greater than 1, the state variables of a jumping cell change into those of its neighboring cell and the grain coarsens. At the same time, the growth length loses its meaning and is set to zero again. Otherwise, the growth distance continues evolving according to eq. (8).

(3) Microstructure evolution. After the above mentioned steps, the state variables of each cell on the boundaries and their neighboring cells are updated, and the microstructural evolution of CA modeling proceeds.

\section{Model calibration}

In this paper, the system is divided into a $500 \times 500$ square lattice and a periodic boundary condition with Neumann neighborhood is adopted. The simulation of grain coarsening by CA models has been carried out on titanium alloys of Ti-6Al-4V, TG6(Ti-5.8Al-4.0Sn-4.0Zr-0.7Nb-1.5Ta-0.4Si$0.06 \mathrm{C}$, wt $\%$ ) and $\mathrm{Ti}-17$ (Ti-5Al-4Mo-4Cr-2Sn-2Zr, wt $\%$ ) in the beta phase field. The predicted coarsening kinetics were compared with experimental observations.

\subsection{Application to Ti-6Al-4V alloy}

$\mathrm{Ti}-6 \mathrm{Al}-4 \mathrm{~V}$ is a two-phase titanium alloy. Its chemical composition is listed in Table 1 . Table 2 shows the material parameters used in eq. (10). From eq. (10), the diffusion velocity of each type of solute atom (including impurities) in Ti-6Al-4V alloy could be obtained; these values are listed in Table 3 .

According to above results (Table 3), the diffusion velocity of solute atoms including $\mathrm{Al}$ and impurity atoms such

Table 1 The chemical composition of Ti-6Al-4V titanium alloy [33]

\begin{tabular}{ccccccccc}
\hline $\begin{array}{c}\text { Solute } \\
\text { elements }\end{array}$ & $\mathrm{Al}$ & $\mathrm{V}$ & $\mathrm{Fe}$ & $\mathrm{C}$ & $\mathrm{O}$ & $\mathrm{N}$ & $\mathrm{H}$ & $\mathrm{Ti}$ \\
\hline $\begin{array}{c}\text { Fraction } \\
(\mathrm{wt} \%)\end{array}$ & 6.1 & 4.0 & 0.1 & 0.21 & 0.13 & 0.005 & 0.004 & Balanced \\
\hline
\end{tabular}

Table 2 The radius and mass of solute atoms in Ti-6Al-4V titanium alloy

\begin{tabular}{ccccccccc}
\hline Atoms & $\mathrm{Ti}$ & $\mathrm{Al}$ & $\mathrm{V}$ & $\mathrm{Fe}$ & $\mathrm{C}$ & $\mathrm{O}$ & $\mathrm{N}$ & $\mathrm{H}$ \\
\hline $\begin{array}{c}\text { Radius } \\
(\mathrm{pm})\end{array}$ & 140 & 125 & 135 & 140 & 70 & 60 & 65 & 21 \\
Mass & 47.87 & 27 & 51 & 56 & 12 & 16 & 14 & 1 \\
\hline
\end{tabular}

Table 3 The ratio of diffusion velocities of solute atoms to Ti in single field

\begin{tabular}{cccccccc}
\hline Solute atoms & $\mathrm{Al}$ & $\mathrm{V}$ & $\mathrm{Fe}$ & $\mathrm{C}$ & $\mathrm{O}$ & $\mathrm{N}$ & $\mathrm{H}$ \\
\hline $\begin{array}{c}\text { Ratio } \\
\left(v_{i} / v_{\mathrm{Ti}}\right)\end{array}$ & 1.98 & 0.97 & 0.855 & 7.98 & 6.98 & 7.36 & 319 \\
\hline
\end{tabular}


as $\mathrm{C}, \mathrm{N}, \mathrm{O}$ and $\mathrm{H}$ is much larger than that of Ti. This means that the presence of atoms on boundaries hardly retards migration of grain boundaries during coarsening, that is, the drag effect is negligible. While the Fe atoms on the boundaries might retard boundary migration because of their smaller diffusion velocity, the volume fraction is so low that the drag effect is also negligible. The diffusion velocity of $\mathrm{V}$ atoms is close to that of $\mathrm{Ti}$, which also results in little effect of solute atoms on grain coarsening. Overall, the drag effects induced by solute and impurity atoms on boundary migration are negligible, so that the static grain coarsening for $\mathrm{Ti}-6 \mathrm{Al}-4 \mathrm{~V}$ alloy could be considered to be an ideal process, as confirmed by Gil's experimental results. Recently, Gil et al. [33] have studied the static coarsening kinetics of $\mathrm{Ti}-6 \mathrm{Al}-4 \mathrm{~V}$ at different experimental temperatures
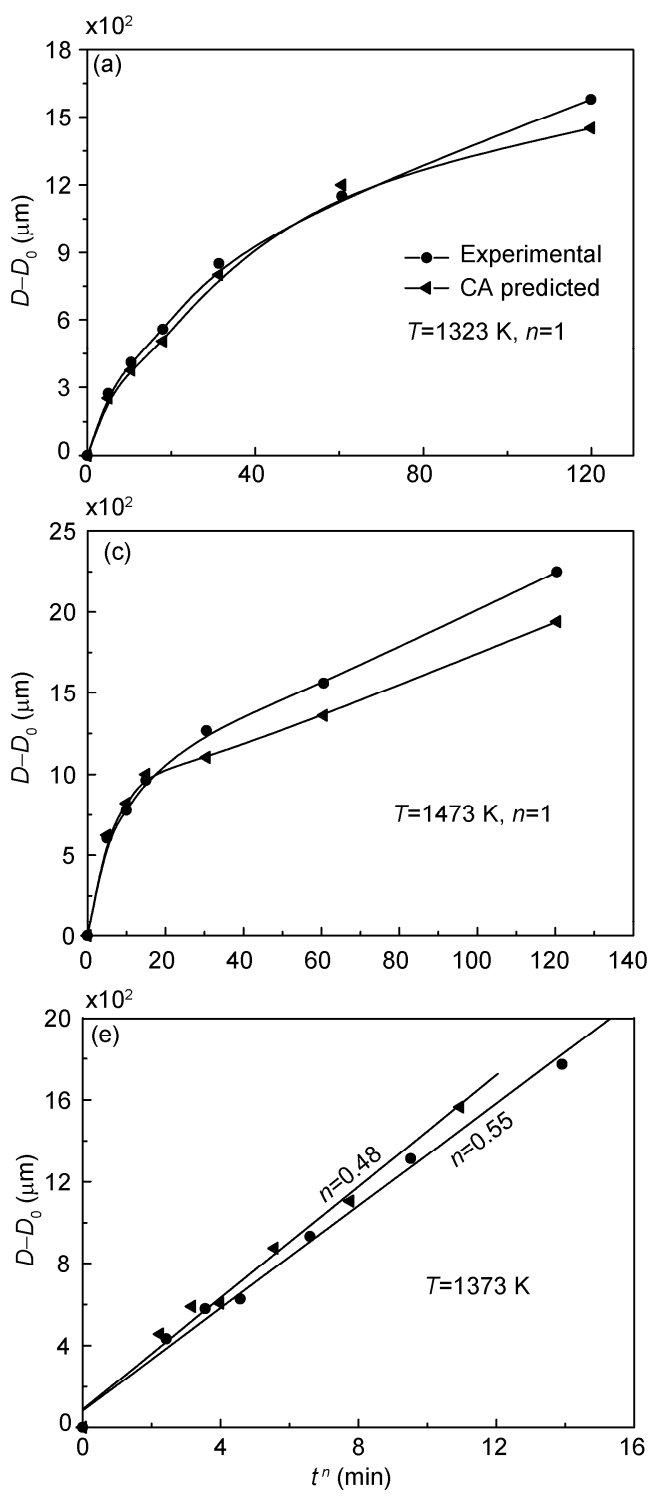

and with different holding times. They concluded that the growth exponent varied from 0.53 to 0.55 , close to the ideal value of 0.5 . Thus the static coarsening process for $\mathrm{Ti}-6 \mathrm{Al}-4 \mathrm{~V}$ in a single field is a pure metal coarsening.

In this paper, the CA models including the solute drag and anisotropic mobility were applied to simulate the static grain coarsening of $\mathrm{Ti}-6 \mathrm{Al}-4 \mathrm{~V}$ alloy in a single field. The model parameters including self-diffusion activation energy, grain size of initial microstructure, temperatures and time are taken as the same as those in Gil's work. Figure 2 (a)-(c) shows the relationships between grain size during coarsening and time, obtained from both experimental and CA prediction. The corresponding linear regions are shown in Figure 2 (d)-(f).

As expected, the simulated results show that the growth
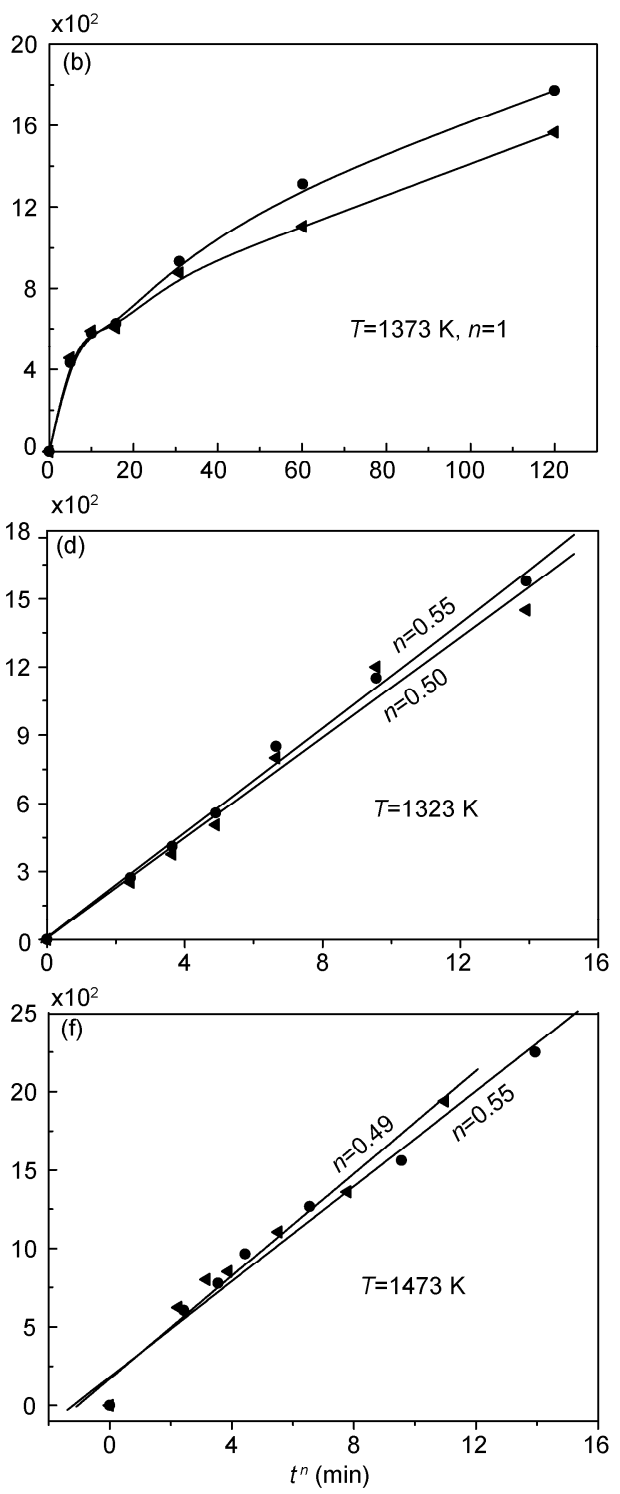

Figure 2 Comparison of experimental and CA predicted static coarsening kinetics for Ti-6Al-4V in single field. (a)-(c) describe the relationship of coarsening grain size and time at 1323,1373 and $1473 \mathrm{~K}$, respectively, $n=1 ;(\mathrm{d})-(\mathrm{e})$ describe the linear relationship of grain size and time at 1323,1373 and 1473 $\mathrm{K}$, respectively. 
rate increases with increasing temperature, which is indicated by the change of slope of curves from Figure 2(a) to (c). The predicted grain sizes indicate very rapid grain growth during the initial stages, while the growth rate gradually drops with increasing time. This may be because of the decrease in the grain boundary area per unit volume, which, in consequence, results in a decrease in the interfacial energy per unit volume [33]. Therefore, the driving force for growth becomes lower and produces a slower kinetic process. However, there are still some differences between the experiments and CA simulations. This may be caused by the limitations of the model, which will be discussed in Section 3.3. A comparison of experimental and simulated coarsening exponents is shown in Table 4.

\subsection{Application to the TG6 and Ti17 alloys}

TG6 alloy (Ti-5.8Al-4.0Sn-4.0Zr-0.7Nb-1.5Ta-0.4Si$0.06 \mathrm{C}, \mathrm{wt} \%$ ) is a new type of near alpha high-temperature titanium alloy, which has been developed for aircraft engine applications [3]. Ti17 alloy (Ti-5Al-4Mo-4Cr-2Sn-2Zr, $\mathrm{wt} \%$ ) is a near beta titanium alloy. Wang et al. [3] have experimentally investigated the static coarsening kinetics for these alloys in beta phase field under isothermal conditions. They found that the coarsening exponent ranged from $0.35-0.38$ for TG6 alloy and $0.27-0.30$ for Ti17 alloy. They also calculated the activation energies of grain coarsening for these alloys. For the TG6 alloy, the value of activation energy ranges from 120.4 to $212.5 \mathrm{~kJ} / \mathrm{mol}$, while for Ti17 alloy, it is from 290.5 to $378.5 \mathrm{~kJ} / \mathrm{mol}$. These values, including coarsening exponent and activation energy, deviate greatly from the ideal values (exponent of 0.5 and activation energy of $97 \mathrm{~kJ} / \mathrm{mol}$ for beta titanium). The drag effect exerted by the solute atoms on the grain boundary may cause the decrease in exponent and increase in activation energy [3].

In this part, the CA model incorporating solute drag and anisotropic mobility is used to simulate the static coarsening of these titanium alloys in the beta field. Their chemical compositions and corresponding material constants are listed in Tables 5 and 6 , respectively. The ratios of solute atom diffusion velocity to $\mathrm{Ti}$ diffusion velocity are calculated according to eq. (10), and the results are listed in Table 7. Figures 3(a)-(c) and 4(a)-(c) show the relationships between grain size and time during coarsening of TG6 and Ti17, respectively; both experimental data and CA predicted data are shown. The corresponding linear regions are shown in Figures 3(d)-(f) and 4(d)-(f), respectively. From these figures, we can see that the predicted values of $n$ agree well with the experimental measurements. The relative errors of $n$ between simulation and experiment are listed in Table 8. The maximum relative error is $13.2 \%$ for TG6 and $20.6 \%$ for Ti17. It is evident that the values are influenced by both the temperature and time.

Table 4 Comparisons of experimental coarsening exponents with those simulated using CA model for Ti-6Al-4V alloy

\begin{tabular}{cccc}
\hline Temperatures $(\mathrm{K})$ & 1323 & 1373 & 1473 \\
\hline Coarsening exponent & $0.55 / 0.50$ & $0.55 / 0.48$ & $0.55 / 0.49$ \\
(experimental/predicted) & 9 & 13 & 11 \\
Relative error $(\%)$ & 9 & 13 & \\
\hline
\end{tabular}

Table 5 The chemical compositions of TG6 and Ti17 alloys (wt\%)

\begin{tabular}{ccccccccccccccc}
\hline Alloy & $\mathrm{Al}$ & $\mathrm{Sn}$ & $\mathrm{Zr}$ & $\mathrm{Mo}$ & $\mathrm{Cr}$ & $\mathrm{Nb}$ & $\mathrm{Ta}$ & $\mathrm{Si}$ & $\mathrm{C}$ & $\mathrm{Fe}$ & $\mathrm{N}$ & $\mathrm{O}$ & $\mathrm{Ti}$ \\
\hline TG6 & 5.74 & 3.94 & 3.78 & - & - & 0.64 & 1.61 & 0.45 & 0.054 & 0.03 & 0.008 & 0.064 & Balanced \\
Ti17 & 5.05 & 2.13 & 2.07 & 4.12 & 4.13 & - & - & - & 0.01 & 0.04 & 0.002 & 0.08 & Balanced \\
\hline
\end{tabular}

Table 6 The radii and masses of solute atoms for TG6 and Ti17 alloys

\begin{tabular}{|c|c|c|c|c|c|c|c|c|c|c|c|c|c|}
\hline Alloy & $\mathrm{Al}$ & $\mathrm{Sn}$ & $\mathrm{Zr}$ & Mo & $\mathrm{Cr}$ & $\mathrm{Nb}$ & $\mathrm{Ta}$ & $\mathrm{Si}$ & $\mathrm{C}$ & $\mathrm{Fe}$ & $\mathrm{N}$ & $\mathrm{O}$ & $\mathrm{Ti}$ \\
\hline Radius (pm) & 125 & 145 & 155 & 145 & 140 & 145 & 145 & 110 & 70 & 140 & 65 & 60 & 140 \\
\hline Mass & 27 & 119 & 91 & 96 & 52 & 93 & 181 & 28 & 12 & 56 & 14 & 16 & 47.87 \\
\hline
\end{tabular}

Table 7 The ratios of diffusion velocities between solute atoms and Ti for TG6 and Ti17 alloys in single field

\begin{tabular}{ccccccccccccc}
\hline Alloy & $\mathrm{Al}$ & $\mathrm{Sn}$ & $\mathrm{Zr}$ & $\mathrm{Mo}$ & $\mathrm{Cr}$ & $\mathrm{Nb}$ & $\mathrm{Ta}$ & $\mathrm{Si}$ & $\mathrm{C}$ & $\mathrm{Fe}$ & $\mathrm{N}$ & $\mathrm{O}$ \\
\hline Ratio $\left(v_{i} / v_{\mathrm{Ti}}\right)$ & 1.98 & 0.388 & 0.475 & 0.48 & 0.92 & 0.497 & 0.255 & 2.18 & 7.97 & 0.855 & 7.36 & 6.98 \\
\hline
\end{tabular}

Table 8 Comparisons of experimental and predicted coarsening exponents for TG6 and Ti17 alloys at different temperatures

\begin{tabular}{|c|c|c|c|c|c|c|}
\hline Temperatures (K) & 1323 & 1423 & 1473 & 1178 & 1188 & 1198 \\
\hline Coarsening exponent & & TG6 & & & Ti17 & \\
\hline (experimental/predicted) & $0.35 / 0.35$ & $0.37 / 0.34$ & $0.38 / 0.33$ & $0.27 / 0.30$ & $0.29 / 0.35$ & $0.30 / 0.35$ \\
\hline Relative error (\%) & 0 & 8 & 13.2 & 11.1 & 20.6 & 16.7 \\
\hline
\end{tabular}



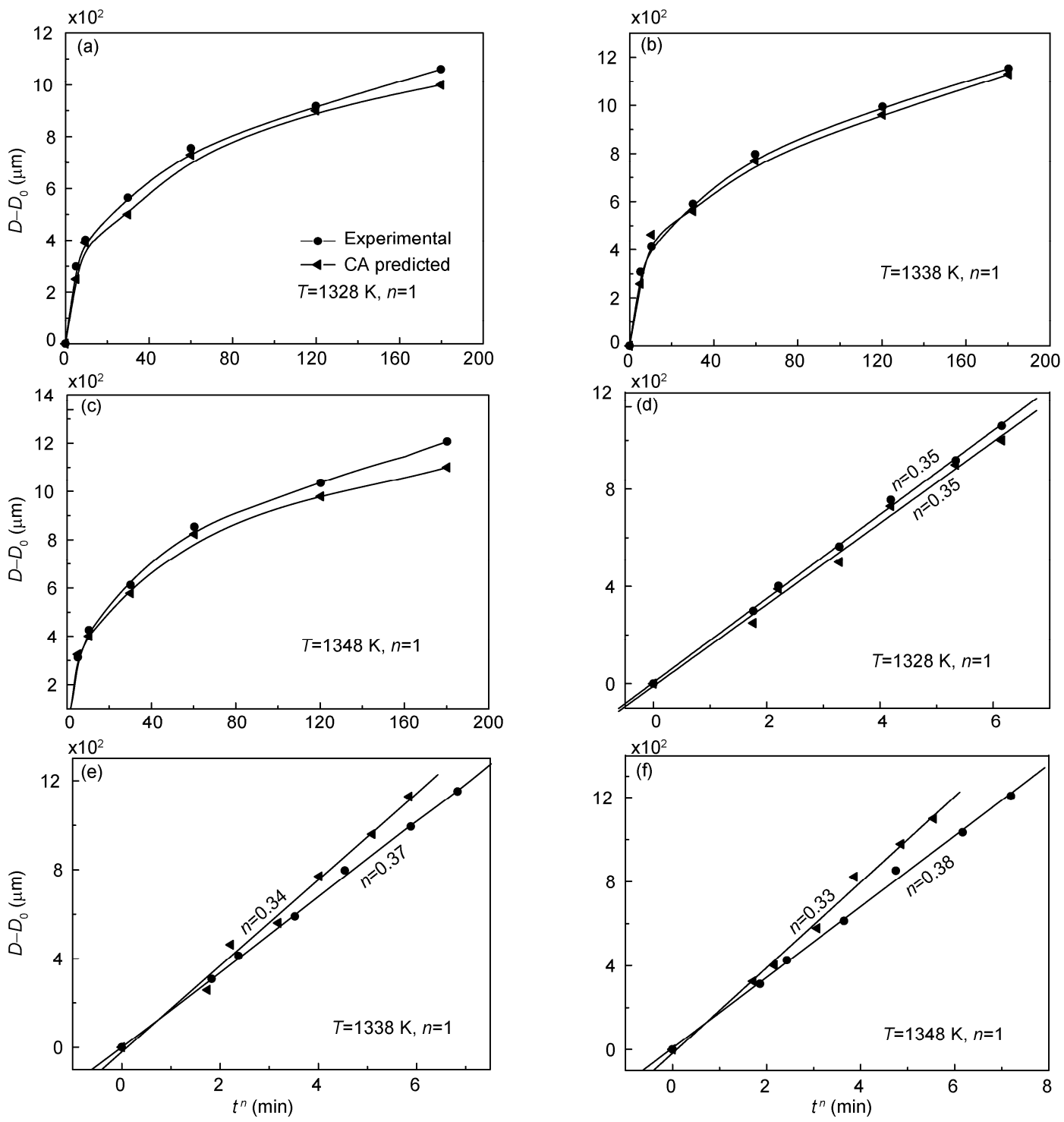

Figure 3 Comparison of experimental and CA predicted coarsening kinetics for TG6 in single field. (a)-(c) describe the relationship of coarsening grain size and time at 1328, 1338 and $1348 \mathrm{~K}$, respectively, $n=1$; (d)-(e) describe the linear relationship of grain size and time at 1328, 1338 and $1348 \mathrm{~K}$, respectively.

\section{Results and discussion}

\subsection{Effect of temperature and time on microstructure}

Figure 5 compares typical microstructures obtained from CA simulation (Figure 5(a)-(c)) and experiment (Figure 5(d)-(f)) for different coarsening times. It is evident that the holding time exerts a significant influence on coarsening. The average grain size increases with increasing time or temperature. Figure 5(a)-(c) shows the microstructure evolution of Ti-6Al-2Zr-1Mo-1V (TA15) alloy at $1313 \mathrm{~K}$ from 10 to $30 \mathrm{~min}$. The predicted grain size (diameter) increases from about 300 to $600 \mu \mathrm{m}$ in this time. The corresponding experimental microstructure evolution for TA15 is shown in Figure 5(d)-(f). The experimental grain size increases from 410 to $630 \mu \mathrm{m}$. The average grain size is larger than the simulated value for the first $10 \mathrm{~min}$, as is shown by comparing Figure 5(a) and (d). The difference may be due to the instability of the initial microstructure where the number of grains changes greatly with holding time. With increasing time, the relative error in grain size between experiment and simulation becomes smaller as the grain number becomes relatively stable. However, the topological evolution of microstructure is hardly affected by time and temperature during coarsening. It is found that the growth of grains with more than six sides is at the cost of grains with less than six sides in these figures, as also shown by Raghavan et al. [22,23] and Chen et al. [26]. This agrees well with von Neumann-Mullins theory that the growth rate of an individual grain strongly depends on the number of grain sides and remains invariant with time or temperature. The 

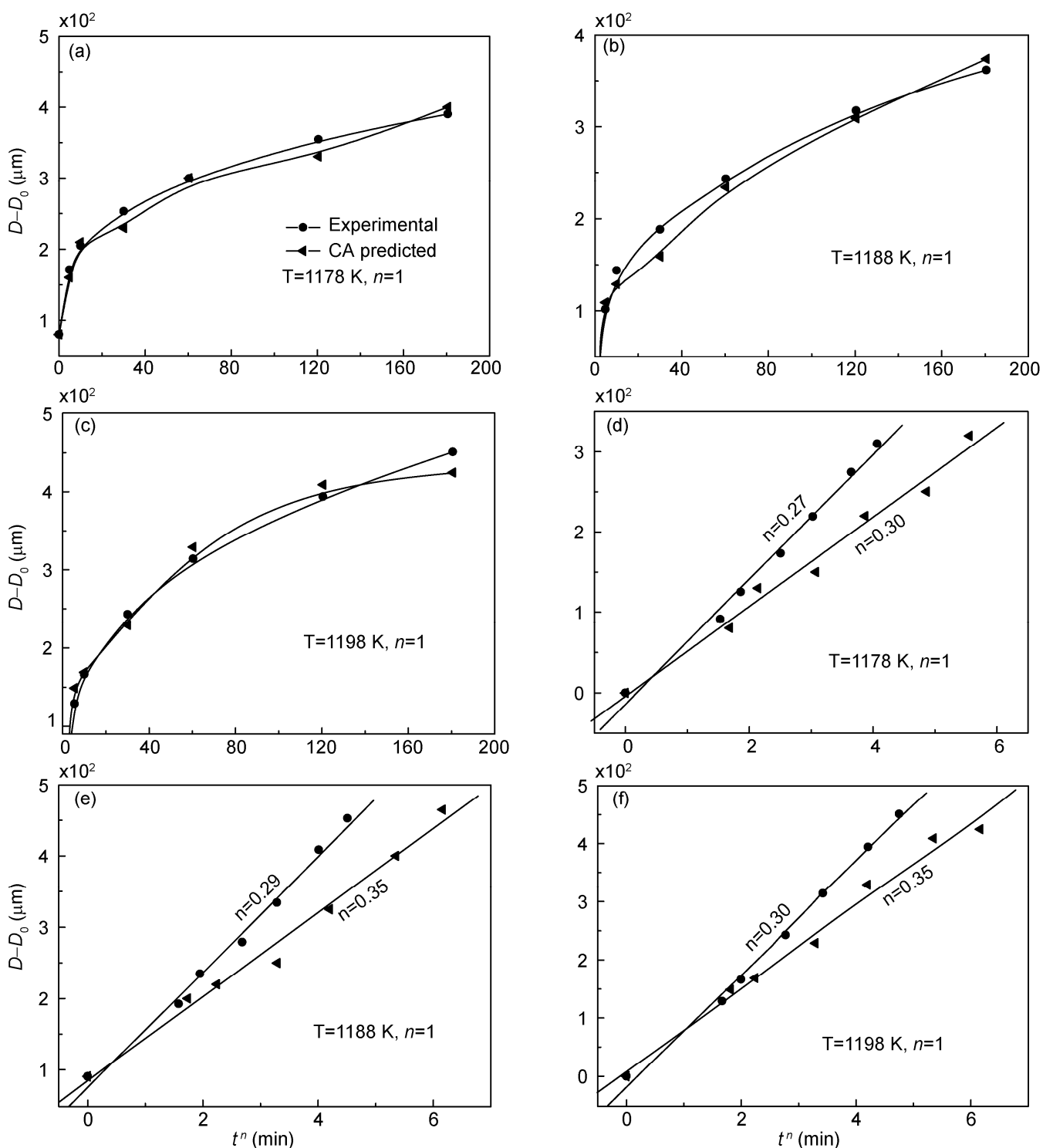

Figure 4 Comparison of experimental and CA predicted coarsening kinetics for Ti-17 in single field. (a)-(c) describe the relationship of coarsening grain size and time at 1178, 1188 and $1198 \mathrm{~K}$, respectively, $n=1$; (d)-(f) describe the linear relationship of grain size and time at 1178,1188 and $1198 \mathrm{~K}$, respectively.

grain side distribution during coarsening keeps a normal trend and remains self-similar (time-invariant). Grain side distribution is an important topology index which has been investigated previously $[11,23]$. Because of statistical errors or the intrinsic disadvantages of the CA method, the predicted side distribution deviates, more or less, from the experimental observation, but is not discussed in this paper.

\subsection{Effect of chemical composition on coarsening kinetics}

The chemical composition significantly influences the static coarsening kinetics. In a single phase field, the coarsening exponent for Ti-6Al-4V alloy ranges from 0.5 to 0.55 [33], which is close to the ideal value 0.5 . But for TG6 and Ti17 alloys, the values of coarsening exponent are much smaller than those of Ti-6Al-4V titanium alloy. This is caused by the solute drag effect. For Ti-6Al-4V alloy, the solute atoms are mainly $\mathrm{Al}$ and $\mathrm{V}$. The diffusion velocity of $\mathrm{Al}$ atoms is nearly equal to that of $\mathrm{Ti}$ (the ratio between them is 0.97 ), and the diffusion velocity of $\mathrm{V}$ atoms is larger than the $\mathrm{Ti}$ diffusion velocity (the ratio between them is 1.98). This means that these two kinds of solute atoms barely retard the movement of grain boundaries during coarsening. Although the diffusion velocity of the impurity $\mathrm{Fe}$ atoms is lower than that of $\mathrm{Ti}$, their volume fraction is so low that the drag effect is also negligible. Therefore, the coarsening exponent for this alloy is close to the ideal value 0.5 .

In comparison, a much larger effect of solute drag on coarsening exponent of TG6 and Ti17 alloys is evident. 

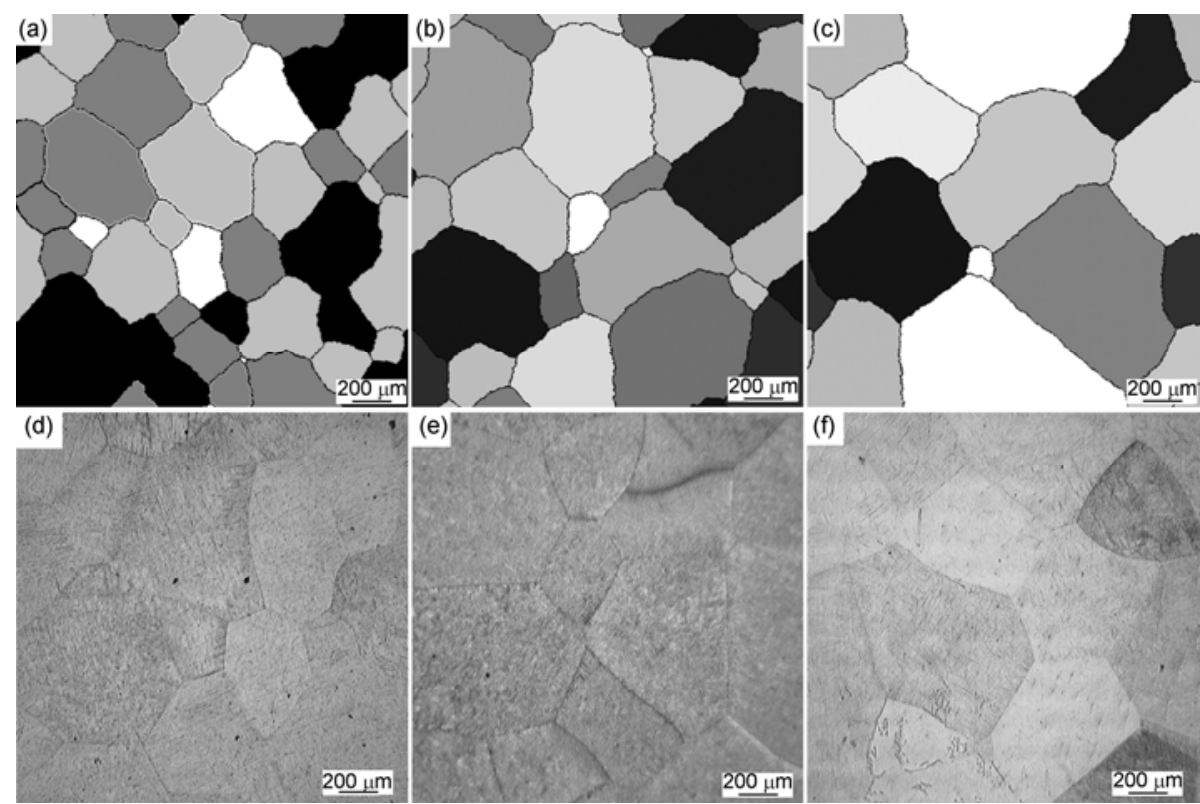

Figure 5 Predicted (a)-(c) and experimental (d)-(f) microstructures of TA15 at $1313 \mathrm{~K}$ with different holding time: (a), (d) $10 \mathrm{~min}$; (b), (e) $30 \mathrm{~min}$; (c), (f) $60 \mathrm{~min}$.

From Table 6, we can see that the radii and masses of solute atoms, such as $\mathrm{Sn}, \mathrm{Zr}, \mathrm{Mo}, \mathrm{Cr}, \mathrm{Nb}$ and $\mathrm{Ta}$, are much larger than those of Ti. Therefore, according to eqs. (10)-(12), the diffusion velocity ratio of these solute atoms to $\mathrm{Ti}$ is less than 1, as shown in Table 7. This means that the solute atoms will retard the boundary coarsening and cause a significant drag effect. Therefore, the predicted values of coarsening exponent for these alloys in a single phase field are in the range of 0.27 to 0.38 , which are smaller than the ideal value 0.5 and agrees well with the experimental values.

\subsection{Limitations of model}

In this paper, a new CA model considering the solute drag effect and anisotropic grain boundary mobility was developed to simulate the static coarsening of titanium alloys in the beta field. This model could be used to predict the effect of temperature and holding time on grain coarsening. During the process, the variation of coarsening kinetics and grain size with temperatures and time was investigated by the CA model. Compared to earlier models, the predicted results agree better with experimental measurements. Alternative factors such as strain and strain rate could also greatly influence grain coarsening. Strain induced by previous deformation or during dynamic coarsening enhances the total energy of each grain, and can change the coarsening kinetics or coarsening exponent. The change of strain rates during dynamic coarsening could influence the coarsening process. Semiatin et al. [8] have studied the dynamic coarsening of Ti-6Al-4V alloy experimentally, and obtained some conclusions. However, in this paper, the CA model mainly simulates the static coarsening phenomenon, where there is no plastic deformation before this process.
Therefore, we do not consider the effect of strain and strain rate on coarsening. The study of dynamic coarsening for duplex titanium alloys in single and two-phase fields by CA modeling will be our next work.

For validation, the CA model was used to simulate the static coarsening of titanium alloys Ti-6Al-4V, TA15, Ti17 and TG6. The comparisons of predicted with experimental results showed good agreement. However, the error is obvious. The average relative errors of coarsening exponent between simulated and experimental values range from $11 \%$ to $13 \%$, and the largest relative error is up to $20.6 \%$ for Ti17 alloy at temperature $1188 \mathrm{~K}$. There are several factors producing these differences between simulated and experimental results. The first is the intrinsic disadvantage of the cellular automaton algorithm. Errors will inevitably be introduced when a complicated continuous process is dealt with by discrete method such as the CA algorithm. The second factor causing errors is the model for ratio of diffusion velocities of solute atoms to that of Ti. This is the largest contributor to the simulated errors. A more accurate and complex formula to describe the diffusion velocity ratio may be needed, which will require many experiments to develop, and may be part of our future work. Thirdly, other factors influencing grain coarsening such as the secondphase particles, precipitation and various microstructural defects are not considered, which also create simulated errors.

\section{Conclusions}

In this paper, a new cellular automaton model, which includes the effects of solute drag and anisotropic grain 
boundary mobility on coarsening, was developed to simulate static grain coarsening. To quantitatively describe the drag effects produced by different solute atoms on coarsening, the diffusion velocities of alloy elements were calculated relative to that of Ti. A formula was proposed to describe the ratio of diffusion velocity between $\mathrm{Ti}$ and solute atoms. Some factors influencing the diffusion velocity such as atomic radius, mass and lattice type were considered. The parameter $c_{0}$ was introduced into the calculation of boundary mobility to consider anisotropy of grain boundaries. The CA models were used to simulate the grain coarsening of Ti-6Al-4V, Ti17, TA15 and TG6 titanium alloys, and the predicted results were in good agreement with experiments.

This work was supported by the National Natural Science Foundation of China (50935007), the National Basic Research Program of China (2010CB731701), the Research Fund of the State Key Laboratory of Solidification Processing (NWPU) (27-TZ-2010) and the "111" Project (B08040).

1 Eylon D, Hall S A, Pierce C M, et al. Microstructure and mechanical properties relationships in the Ti-11 alloy at room and elevated temperatures. Metall Mater Trans A, 1976, 7: 1826-1871

2 Zhang M, Zhang J, McDowell D L. Microstructure-based crystal plasticity modeling of cyclic deformation of Ti-6Al-4V. Int J Plasticity, 2007, 23: 1328-1348

3 Wang T, Guo H Z, Tan L J, et al. Beta grain growth behaviour of TG6 and Ti17 titanium alloys. Mater Sci Eng A, 2011, 528: 6375-6380

4 Ivasishin O M, Shevchenko S L, Semiatin S L. Effect of crystallographic texture on the isothermal beta grain-growth kinetics of Ti-6Al-4V. Mater Sci Eng A, 2002, 332: 343-350

5 Ivasishin O M, Semiatin S L, Markovsky P E, et al. Grain growth and texture evolution in Ti-/6Al-4V during beta annealing under continuous heating conditions. Mater Sci Eng A, 2002, 337: 88-96

6 Semiatin S L, Fagin P N, Glavicic M G, et al. Influence on texture on beta grain growth during continuous annealing of Ti-6Al-4V. Mater Sci Eng A, 2001, 299: 225-234

7 Semiatin S L, Kirby B C, Salishchev G A. Coarsening behavior of an alpha-beta Titanium alloy. Metall Mater Trans A, 2004, 35: 2809-2819

8 Semiatin S L, Corbett M W, Fagin P N, et al. Dynamic-coarsening behavior of an alpha/beta Titanium alloy. Metall Mater Trans A, 2006, 37: 1125-1136

9 Bradley J R, Rigsbee J M, Aaronson H I. Growth kinetics of grain boundary ferrite allotriomorphs in Fe-C alloys. Metall Mater Trans A, 1977, 6: 323-333

10 Cotrina E, Iza-Mendia A, López B. Study of the ferrite grain coarsening behind the transformation front by electron backscattered diffraction techniques. Metall Mater Trans A, 2004, 35: 93-102

11 Tong M M, Li D Z, Li Y Y. Modeling the austenite-ferrite diffusive transformation during continuous cooling on a mesoscale using Monte Carlo method. Acta Mater, 2004, 52: 1155-1162

12 Tong M M, Ni J, Zhang Y, et al. Temporal oscillatory behavior in deformation induced ferrite transformation in an Fe-C binary system. Scripta Mater, 2004, 50: 909-913

13 Song X Y, Liu G Q, Gu N J. A new Monte Carlo simulation of three-dimensional microstructures and their evolution in polycrystal- line. Chin Sci Bull, 1999, 44: 1432-1436

14 Luo B C, Wang H P, Wei B B. Phase field simulation of monotectic transformation for liquid Ni-Cu-Pb alloys. Chin Sci Bull, 2009, 54: $183-188$

15 Zaeem M A, Kadiri H El, Wang P T, et al. Investigating the effects of grain boundary energy anisotropy and second-phase particles on grain growth using a phase-field model. Comput Mater Sci, 2011, 50: 2488-2492

16 Zheng C W, Xiao N M, Li D Z, et al. Microstructure prediction of the austenite recrystallization during multi-pass steel strip hot rolling: A cellular automaton modeling. Comput Mater Sci, 2008, 44: 507-514

17 Zheng C W, Xiao N M, Li D Z. Mesoscopic modeling of austenite static recrystallization in a low carbon steel using a coupled simulation method. Comput Mater Sci, 2009, 45: 568-575

18 Ding R, Guo Z X. Microstructural modeling of dynamic recrystallization using an extended cellular automaton approach. Comput Mater Sci, 2002, 23: 209-218

19 Yang H, Wu C, Li H W, et al. Review on cellular automata simulations of microstructure evolution during metal forming process: Grain coarsening, recrystallization and phase transformation. Sci China Tech Sci, 2011, 54: 2107-2118

20 Ding H L, He Y Z, Liu L F. Cellular automata simulation of grain growth in three dimensions based on the lowest-energy principle. J Cry Grow, 2006, 293: 489-497

21 Geiger J, Roósz A, Barkóczyb P. Simulation of grain coarsening in two dimensions by cellular automaton. Acta Mater, 2001, 49: 623-629

22 Raghavan S, Sahay S S. Modeling the grain growth kinetics by cellular automaton. Mater Sci Eng A, 2007, 445-446: 203-209

23 Raghavan S, Sahay S S. Modeling the topological features during grain growth by cellular automaton. Comput Mater Sci, 2009, 46: 92-99

24 Kugler G, Turk R. Study of the influence of initial microstructure topology on the kinetics of static recrystallization using a cellular automata model. Comput Mater Sci, 2006, 37: 284-291

25 Mao W M, An Z G, Li S X. Influence of MnS particles on the behaviors of grain boundary migration in Fe-3\%Si alloys. Chin Sci Bull, 2009, 54: 4537-4540

26 Chen F, Cui Z S, Liu J. Mesoscale simulation of the high-temperature austenitizing and dynamic recrystallization by coupling a cellular automaton with a topology deformation technique. Mater Sci Eng A, 2010, 527: 5539-5549

27 He Y Z, Ding H L, Liu L F, et al. Computer simulation of 2D grain growth using a cellular automata model based on the lowest energy principle. Mater Sci Eng A, 2006, 429: 236-246

28 Yazdipour N, Davies C H J, Hodgson P D. Microstructural modeling of dynamic recrystallization using irregular cellular automata. Comput Mater Sci, 2008, 44: 566-576

29 Chen F, Cui Z S, Liu J. Modeling and simulation on dynamic recrystallization of $30 \mathrm{Cr} 2 \mathrm{Ni} 4 \mathrm{MoV}$ rotor steel using the cellular automaton method. Model Simul Mater Sci Eng, 2009, 17: 1-19

30 Kugler G, Turk R. Modeling the dynamic recrystallization under multi-stage hot deformation. Acta Mater, 2004, 52: 4659-4668

31 Fjeldberg E, Marthinsen K. A 3D Monte Carlo study of the effect of grain boundary anisotropy and particles on the size distribution of grains after recrystallisation and grain growth. Comput Mater Sci, 2010, 48: 267-281

32 Chun Y B, Semiatin S L, Hwang S K. Monte Carlo modeling of microstructure evolution during the static recrystallization of cold-rolled, commercial-purity titanium. Acta Mater, 2006, 54: 3673-3689

33 Gil F J, Planell J A. Behaviour of normal grain growth kinetics in single phase titanium and titanium alloys. Mater Sci Eng A, 2000, 283: $17-24$

Open Access This article is distributed under the terms of the Creative Commons Attribution License which permits any use, distribution, and reproduction in any medium, provided the original author(s) and source are credited. 